\title{
AGRESIONES CONTRA LOS PROFESIONALES DE LA SALUD EN LOS SERVICIOS DE URGENCIAS
}

\author{
Álvaro L. Fajardo Zapata'
}

\section{Resumen}

Objetivo: Identificar las agresiones contra los médicos y enfermeras en los servicios de urgencias por parte de los usuarios del sistema de salud. Materiales y métodos: Estudio descriptivo transversal. Se realizó un muestreo no probabilístico de tipo intencional por unidad hospitalaria tomando en consideración la agrupación por redes de la ciudad. Se aplicó una encuesta voluntaria y personal a médicos (120) y enfermeras (120) de los servicios de urgencias de las cuatro redes públicas de Bogotá. Resultados: El 87,1\% de todos los profesionales de la salud que participaron en el estudio reportaron que fueron objeto de actos violentos por parte de los usuarios durante el desarrollo de sus actividades laborales. El 56,6\% de las agresiones fueron verbales, el 2,1\% físicas y el $28,3 \%$ tanto físicas como verbales. El 90,8\% de los médicos manifestó haber recibido algún tipo de agresión, mientras que el 83,3\% de las enfermeras(os) manifestaron igualmente haber sido objeto de ellas $\left(\mathrm{Chi}^{2}=0,4962 ; \mathrm{p}=0,4811\right)$. Conclusiones: Los profesionales de la salud que laboran en los servicios de urgencias son objeto de permanentes agresiones verbales, físicas o ambas por parte de los usuarios del sistema.

Palabras clave: agresión, servicios médicos de urgencias, violencia, personal de salud, enfermeros.

\footnotetext{
${ }^{1}$ M.Sc. en Salud Pública y Desarrollo Social. Especialista en Salud Ocupacional. Profesor asociado del Departamento de Ingeniería y Ciencias Básicas de la Fundación Universitaria del Área Andina. Bogotá, Colombia. Correo electrónico: afajardo@ areandina.edu.co, afajardo2004@gmail.com
} 


\title{
AGGRESSIONS AGAINST HEALTH PROFESSIONALS IN EMERGENCY SERVICES
}

\author{
Álvaro L. Fajardo Zapata
}

\section{Abstract}

Objective: Identify the aggressions against the doctors and nurses in the emergency services by the users of the health system. Materials and methods: Cross-sectional descriptive study. A non-probabilistic sampling study of an intentional type was carried out per hospital unit taking into account the clustering by city networks. A voluntary and personal survey was applied to physicians (120) and nurses (120) of the emergency services of the four Bogotá public networks. Results: $87.1 \%$ of all health professionals who participated in the study reported that they were subjected to violent acts by users during the development of their work activities. $56.6 \%$ of the attacks were verbal, $2.1 \%$ physical and $28.3 \%$ both physical and verbal. $90.8 \%$ of the physicians reported having received some type of aggression, while $83.3 \%$ of the nurses also reported having undergone them $($ Chi $2=0.4962, \mathrm{p}=0.4811)$. Conclusions: The health professionals who work in the emergency services are subject to permanent verbal, physical or both aggressions by the users of the system. 


\section{AGRESSÕES CONTRA OS PROFISSIONAIS DA SAÚDE NOS SERVIÇOS DE URGÊNCIAS}

Álvaro L. Fajardo Zapata

\section{Resumo}

Objetivo: identificar as agressões contra os médicos e enfermeiros nos serviços de urgências por parte dos usuários do sistema de saúde. Métodos: estudo descritivo-transversal. Realizou-se uma amostragem não probabilístico intencional por unidade hospitaleira, usando as considerações das agrupações por redes nas cidades. Foi usado uma enquete voluntaria e pessoal para os médicos $(\mathrm{N}=120)$, enfermeiros $(\mathrm{N}=120)$, dos serviços de urgências das quatro redes públicas da cidade de Bogotá. Resultados: encontra-se que o $87,1 \%$ dos profissionais da saúde que participaram do estudo reportaram que foram objeto de ações violentas por parte dos usuários durante o desenvolvimento das atividades laborais. Além disso, as agressões apresentam $56,6 \%$ sendo verbais, $2,1 \%$ físicas e $28,3 \%$ combinadas (físicas e verbais). No final, os médicos manifestam no 90,8\% receber alguma forma de agressão, assim o 83,3\% dos enfermeiros tem sido estas agressões (Chi= 0,4962; $\mathrm{p}=0,4811$ ). Conclusões: os profissionais da saúde que trabalham nos serviços de urgências são objeto de permanentes agressões verbais, físicas ou combinadas pelos usuários do sistema. 


\section{Introducción}

La violencia es un fenómeno omnipresente que está enquistado en la sociedad y se puede encontrar en todos los ámbitos, y el lugar de trabajo no es la excepción. La violencia física o psicológica en el ámbito laboral es un problema mundial que afecta a todos los sectores de la economía, y a todas las ocupaciones, donde los trabajadores que están en mayor riesgo son los profesionales del sector salud, los policías y los vigilantes $(1,2)$.

De acuerdo con la Organización Internacional del Trabajo (OIT), la violencia en el lugar de trabajo se define como "toda acción, incidente o comportamiento que se aparta de lo razonable mediante el cual una persona es agredida, amenazada, humillada o lesionada por otra, en el ejercicio de su actividad profesional o como consecuencia directa de la misma” (3). La agresión puede ser física o verbal, donde la violencia física entraña un comportamiento intencionado con el empleo de la fuerza física, la cual puede manifestarse como patadas, bofetadas, puñaladas, tiros, empujones, mordiscos y pellizcos. La agresión verbal es definida como un comportamiento amenazante del uso de la fuerza física o del poder, las humillaciones, los insultos, las injurias, la falta de respeto a la dignidad y al valor de la persona $(4,5)$. La citada Organización, reveló que en 2002 cerca del $25 \%$ de los incidentes violentos en el lugar de trabajo se produjeron en el sector de la salud y más del 50\% de los profesionales de esta disciplina han experimentado inciden-

1784 tes de este tipo. Sumado a lo anterior, el Consejo General de Enfermería de
España en un estudio reveló que el 33\% del personal de enfermería español ha sufrido agresiones físicas o verbales en el último año (6).

Los profesionales del sector de la salud están expuestos a muchos peligros en su ambiente laboral, los cuales van desde insultos verbales hasta la violencia física. Estas agresiones tienen lugar en los diversos servicios y en diversas situaciones dentro de las que se pueden nombrar la negación de un servicio, demora o fallas en la atención, ingreso de pacientes enajenados, ebrios o bajo los efectos de sustancias psicoactivas, nivel cultural y socioeconómico de los usuarios y el funcionamiento del sistema de salud (7). Las agresiones pueden ser realizadas por el paciente, por sus familiares o por los acompañantes. Aunque cualquier persona que labore en una institución de salud podría a llegar a ser víctima de violencia, es el personal que está en contacto directo con los pacientes quien más fácilmente puede ser agredido, tales como los médicos y las enfermeras(os) $(8,9)$.

Las agresiones pueden derivar en heridas físicas de poca gravedad, heridas graves, invalidez temporal o permanente, trauma psicológico o la muerte. Aparte de esto, el personal de salud se ve enfrentado a otros agentes generadores de estrés como lo son el contacto cotidiano con el dolor humano, peticiones excesivas por parte de los usuarios, incapacidad para responder a las demandas de los usuarios o sus familiares $y$, por supuesto, el temor a cometer errores en su práctica clínica $(1,6,10-13)$.

La violencia física y verbal cometida contra los médicos y las enfermeras(os) ha sido 
reportada por varios estudios realizados en España; también se han identificadolas causas generadoras tales como los tiempos de espera, la intoxicación alcohólica por parte del paciente, la presencia de trastornos mentales, la calidad de la información y el trato recibido. Estos estudios han identificado el tipo de agresor, dentro de los que se destacan el propio paciente, el acompañante $\mathrm{o}$ ambos $(11,12,14)$.

Así mismo, en Argentina, Perú, Canadá, Reino Unido y Estados Unidos se han realizado estudios que han evidenciado la agresión contra estos profesionales de la salud. Dichos estudios han reportado que la mayor parte de las agresiones corresponden a agresiones verbales, donde predominan los gritos y las descalificaciones. La mayoría de los estudios han revelado que los profesionales que laboran en los servicios de urgencias son quienes están más expuestos a estos tipos de actos (18-25).

Hay que mencionar, además, que los profesionales que han participado en los estudios han manifestado haber sido agredidos en más de una ocasión. En cuanto al género de los profesionales agredidos, los estudios no apuntan a un género en particular, aunque algunos estudios muestran, que son las enfermeras(os), quienes están más expuestas a sufrir las agresiones $(16,17)$.

La mayoría de las agresiones de que son víctimas los profesionales de la salud no salen a la luz pública porque los profesionales no las denuncian, lo cual puede ser debido a que estos profesionales minimizan el acto violento y no le dan la importancia requerida o, aún más grave, los consideran "normales" o piensan quela denuncia es inútil. La mayoría de los actos conflictivos asistenciales y las agresiones contra el personal de salud suceden en los servicios de urgencias $(2,9,13,22,25)$.

En Colombia, no se encontraron estudios donde se reporten las agresiones contra los profesionales de la salud. A través de la consulta de diarios se encontraron reportes de violencia contra estos profesionales en los siguientes medios: El Colombiano, del 28 junio de 2015 (26); El Espectador, de febrero 24 de 2011 (27); El Tiempo, en su edición del 11 de julio de 2014 (28); el Diario ADN, del 20 de diciembre de 2014 (29); el Diario La Opinión, de Cúcuta, del 24 de junio de 2015 (30).

De acuerdo con lo anterior, es importante señalar que las agresiones de que son víctima los profesionales de salud de los servicios de urgencias por parte de los usuarios del sistema son un problema relevante que no ha sido investigado en Colombia y solo ha sido mostrado someramente por los diarios, pero sin indagar sus razones causales. La presente investigación apunta a caracterizar esta problemática y a establecer la frecuencia, a fin de que pueda ser utilizada para desarrollar estrategias de control.

\section{Materiales y métodos}

Se realizó un estudio epidemiológico observacional transversal, cuyo objetivo fue identificar las agresiones contra los médicos y enfermeras por parte de los usuarios del sistema de salud en los servicios de urgencias de las cuatro redes que agrupan las 22 instituciones públicas de Bogotá: norte, suroccidente, sur, 
centro-oriente. Se realizó un muestreo no probabilístico de tipo intencional por unidad hospitalaria tomando en consideración la agrupación por zonas de la ciudad. Se tomó como error el 9\%, una prevalencia estimada del $50 \%$ y un nivel de confianza del $95 \%$. Se realizó un muestreo por separado, es decir, uno para el personal médico y otro para el personal de enfermería. Se estimó el tamaño de la muestra en 120 encuestas para cada grupo de profesionales. Se diseñó y aplicó un instrumento (encuesta) donde se indagó por edad, género, años de egresado de la profesión, antigüedad en el servicio de urgencias, tipo de agresión de la que fue objeto, frecuencia de agresiones, razones por las que se generó la agresión y tipo de agresor. La encuesta fue sometida a juicio de expertos del área de la salud y fue aplicada por estudiantes, quienes previamente fueron capacitados. La participación en el estudio fue voluntaria, previa firma del consentimiento informado. No se realizó ninguna intervención durante el desarrollo del estudio. Se excluyeron las encuestas que no estuvieran completamente diligenciadas. El manejo de la información fue anónimo y confidencial. El estudio fue aprobado por el comité de ética de la Institución. Los datos producto de la investigación fueron ingresados en una base de datos que se elaboró en Excel y los análisis se realizaron en Epi Info 7. Se realizó un estudio de frecuencias y determinación de prevalencias mediante un análisis univariado. Se planteó una asociación estadística únicamente con el ánimo de profundizar un poco más 1786 en los hallazgos obtenidos, y se aplicó la prueba Chi cuadrado.

\section{Resultados}

El 87,1\% de los profesionales de la salud que participaron en este estudio fue objeto de actos violentos durante el desarrollo de sus actividades laborales. El 56,6\% de las agresiones fueron únicamente verbales, el 2,1\% únicamente físicas y el 28,3\% tanto físicas como verbales. El $90,8 \%$ de los médicos manifestó haber recibido algún tipo de agresión, mientras que el $83,3 \%$ de las enfermeras(os) manifestaron igualmente haber sido objeto de ellas. No se encontró asociación entre la agresión y el ser profesional de la salud $\left(\mathrm{Chi}^{2}=\right.$ 0,$4962 ; \mathrm{p}=0,4811$ ).

Por otro lado, los profesionales que participaron en el estudio manifestaron que las agresiones habían sido reiteradas, ya que el $79,8 \%$ de los médicos manifestaron haber sido agredidos en más de una ocasión y el $80 \%$ del personal de enfermería refirió la misma situación (figura 1).

En cuanto al tipo de agresión, el 59,7\% de los médicos expresaron haber sido agredidos únicamente de forma verbal, mientras que el personal de enfermería lo fue en un 71\%; un 1,5\% de los médicos declaró haber recibido únicamente agresiones físicas, contra un 3\% del personal de enfermería. El 38,5\% de los médicos y del personal de enfermería manifestaron haber sido agredidos tanto verbal como físicamente. No se encontró asociación entre la agresión y el género del profesional de la salud $\left(\mathrm{Chi}^{2}=2,18 ; \mathrm{p}=0,39\right)$ (figura 2).

En cuanto al tipo de agresiones verbales, fueron los gritos e insultos los más 
reportados, seguidos por las amenazas, la descalificación yla humillación (figura 3).

En las agresiones físicas sobresalieron los empujones, el lanzamiento de objetos, los escupitajos y las patadas.

Por otra parte, no todas las agresiones fueron reportadas por los profesionales de salud a la oficina de seguridad y salud en el trabajo, ya que solo el $39,4 \%$ del personal médico y el $12 \%$ del personal de enfermería que fueron agredidos manifestaron haberlo reportado. Asimismo, el 17,4\% de los médicos y el $9 \%$ del personal de enfermería manifestaron que reportaron ante las autoridades correspondientes las agresiones de la cuales fueron objeto (figura 4).

En cuanto a la edad promedio de los agresores, los médicos reportaron que en su mayoría correspondieron a personas que tenían edades que oscilaban entre los 25 a 29 años (49,5\%), mientras que el personal de enfermería reportó que el mayor porcentaje (27\%) también correspondió a este grupo etario. Asimismo, el género masculino fue el mayor agresor del personal médico $(61,8 \%)$, al igual que del personal de enfermería (57,9\%) (figura 5).

El mayor causante de las agresiones tanto al personal médico como al de enfermería correspondió a los familiares del paciente, seguidos por las agresiones proferidas por el mismo paciente.

Se debe agregar también que el 32\% de los médicos y el 34,2\% del personal de enfermería consideran que las agresiones son normales en el desarrollo de sus actividades laborales.

\section{Discusión}

La violencia física o verbal a que son sometidos los médicos y las enfermeras en el ámbito laboral ha sido evidenciada por varios estudios en el mundo. Los hallazgos de violencia contra los profesionales de la salud en los servicios de urgencias de la red pública de Bogotá del presente estudio $(87,1 \%)$ están por encima a los reportados por otros estudios, tales como España por Cervantes et al. (14), Ortells et al. (15), Galián et al. (11), Bernaldo-de-Quirós et al. (17) y el observatorio de agresiones de la Comunidad de Castilla y León; así mismo, en Estados Unidos, por Kowalenko et al. (22); en Argentina, por Farias et al. (18), y Travetto et al. (19); por Winstanle, en el reino Unido; por Ancalli et al. (23) y Tuya et al. (24), en Perú. Sin embargo, están por debajo de los encontrados por Miedema et al. (20), en Canadá, quienes en médicos familiares encontraron que el 98\% de los encuestados manifestó haber sido objeto de al menos un incidente de abuso.

Hay que mencionar, además, que en el presente estudio se encontró que los médicos son más agredidos que las enfermeras, datos que no coinciden con los de Lameiro et al. (16), en España, quienes encontraron que los profesionales más afectados por este fenómeno social fueron las enfermeras(os).

En cuanto al tipo de agresión, este estudio encontró que el 56,6\% de los profesionales recibieron únicamente agresiones verbales, el 2,1\% solo físicas y el 28,3\% manifestó haber sido agredidos tanto física como verbalmente, porcentajes que son 


\section{Agresiones a profesionales de la salud}

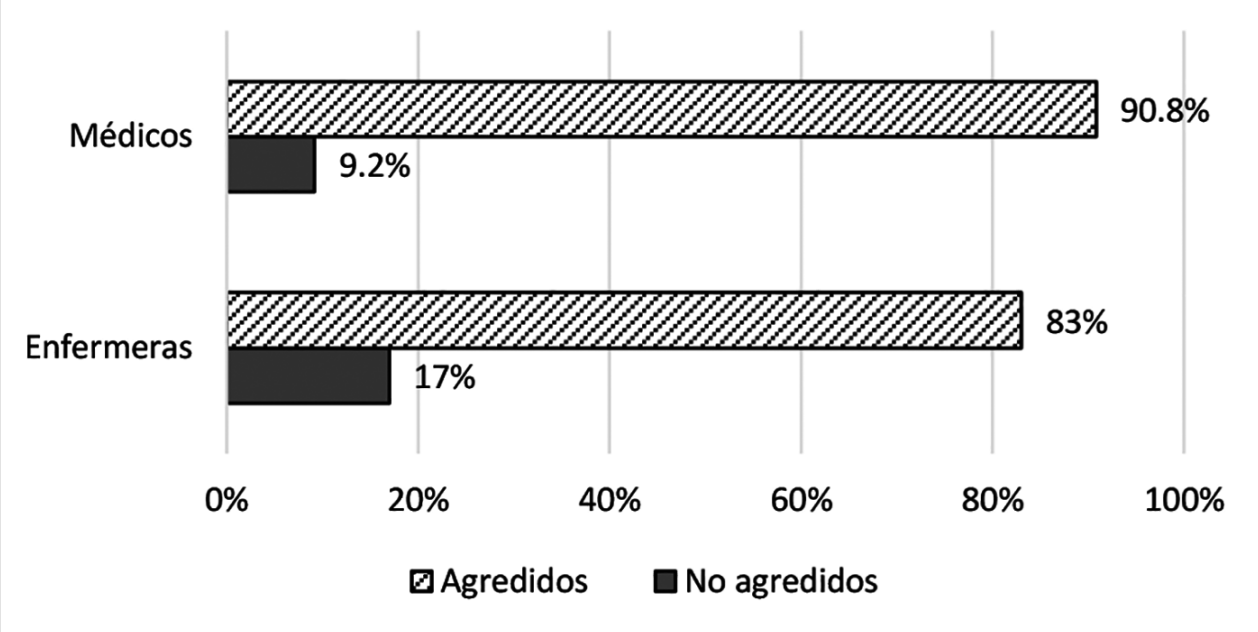

Figura 1. Agresiones a profesionales de la salud

Fuente: Elaboración propia.

\section{Tipos de agresiones verbales}

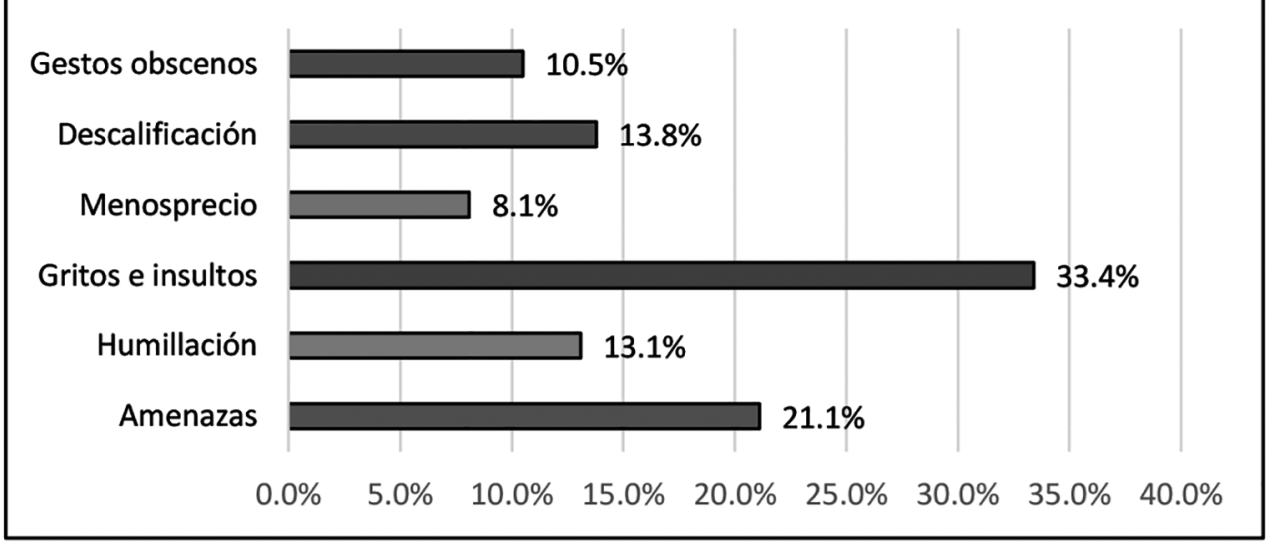

Figura 2. Tipos de agresiones verbales

Fuente: Elaboración propia. 


\section{Tipos de agresiones físicas}

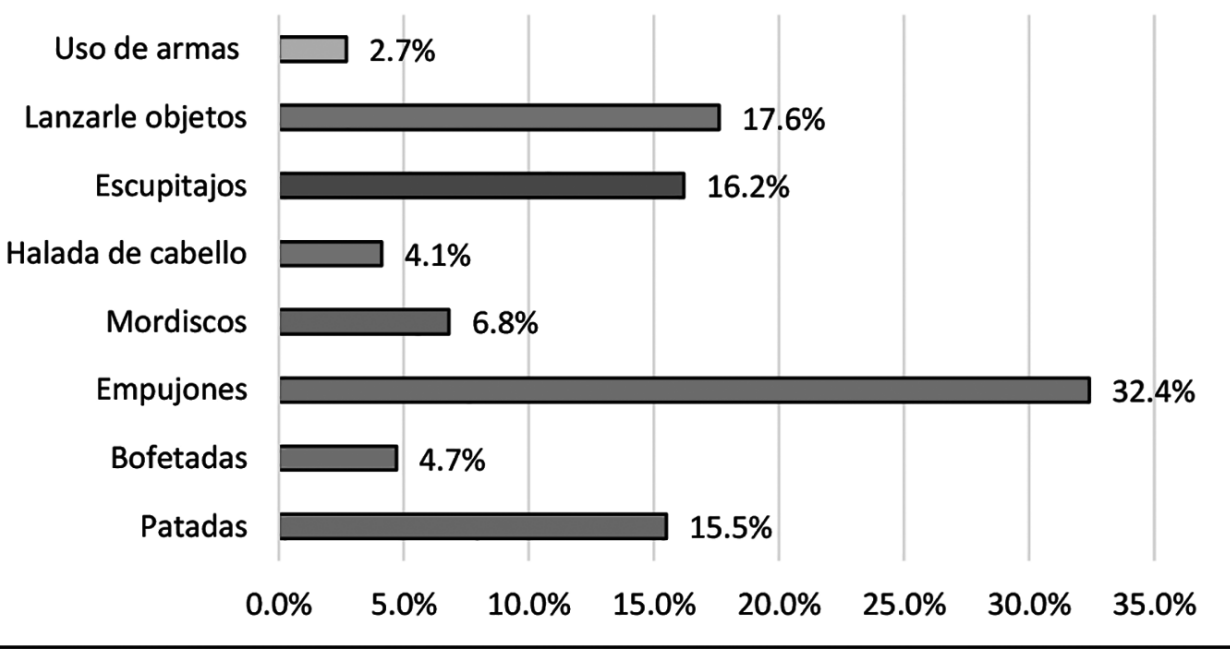

Figura 3. Tipos de agresiones físicas

Fuente: Elaboración propia.

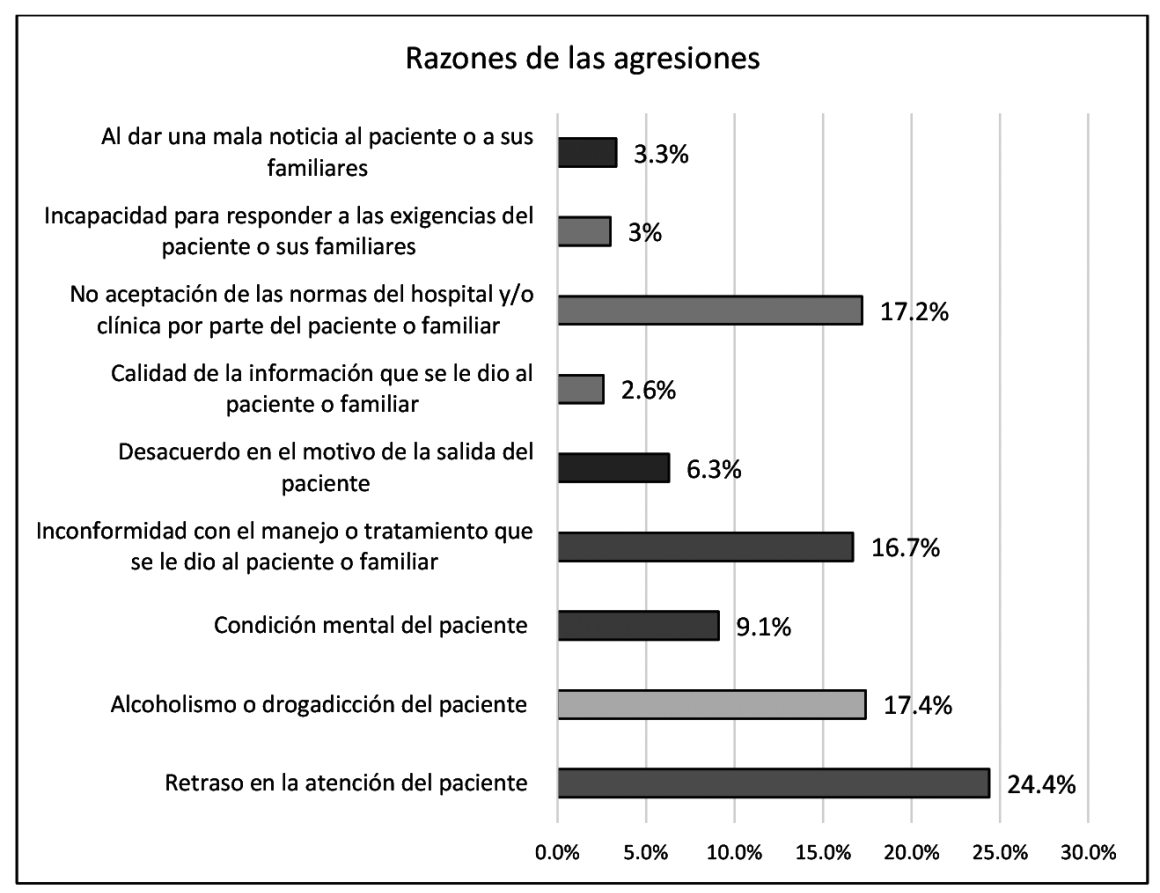

Figura 4. Razones de las agresiones.

Fuente: Elaboración propia. 


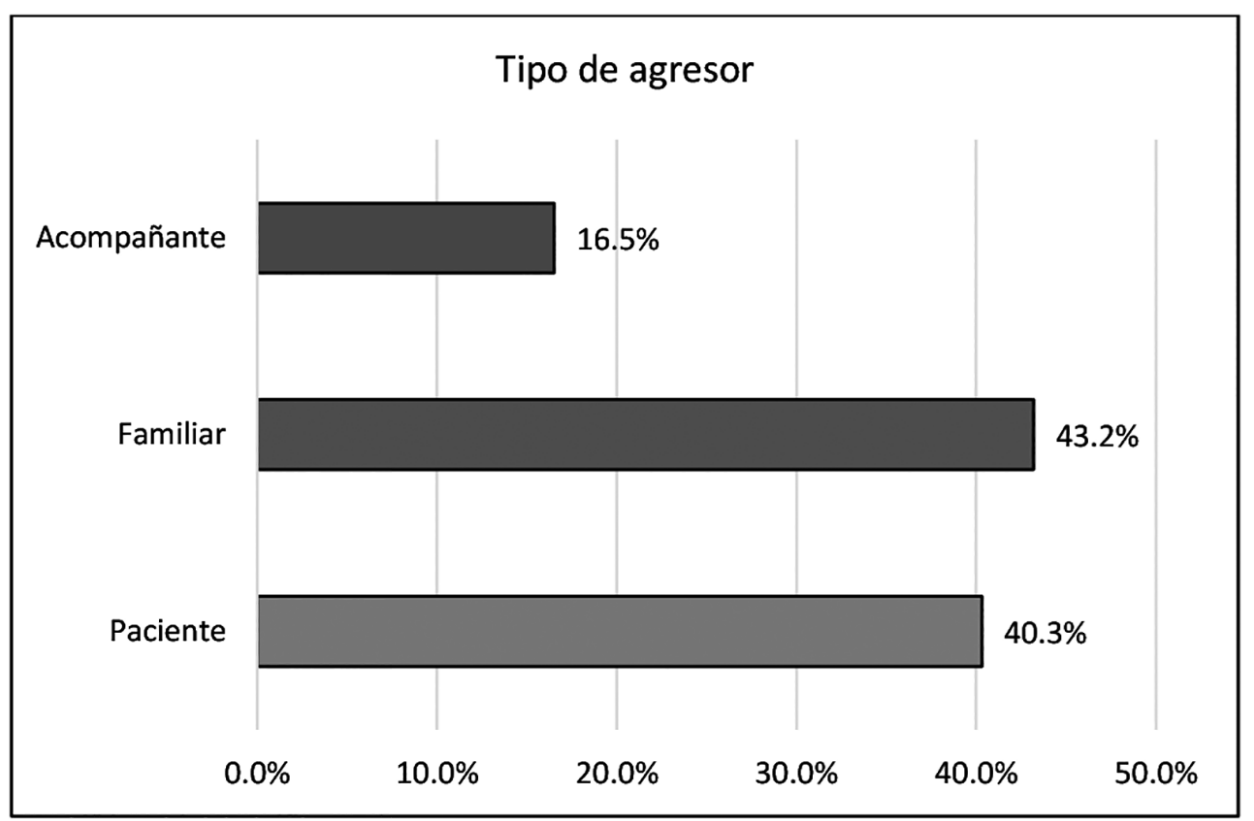

Figura 5. Tipo de agresor

Fuente: Elaboración propia.

inferiores a los reportados por Lameiro et al. (16), quienes reportaron niveles de agresión física del $80 \%$ y verbales con un 20\%; Martínez et al. (12), quienes encontraron un $64 \%$ de violencia psicológica, y Farias et al. (18), con un 54,2\%.

Los niveles de violencia verbal encontrados en este estudio coinciden con los de Bernaldo-de-Quirós et al. (17), en España, quienes reportaron que la violencia verbal oscilaba entre el $60,6 \%$ y el $76,4 \%$, Miedema et al. (20), en Canadá (75\%), Kowalenko et al. (22) (75\%); Winstanle (21) (68\%). Niveles superiores de violencia verbal en comparación con los hallazgos de este estudio se encontraron en los estudios de Cervantes et al. (14), quienes hallaron que el $37,9 \%$ correspondió a violencia fí-
En cuanto a las agresiones físicas los hallazgos de este estudio son superiores a los hallazgos de Travetto et al. (19), en Argentina; a las de Ortells et al. (15), y Martínez (12) et al., en España; a las de Winstanle (21), en el Reino Unido; a las de Kowalenko et al. (22), en Estados Unidos, y a las de Tuya et al. (24), en Perú; pero son inferiores a los reportados por Cervantes et al. (14), Lameiro et al. (16), y Bernaldo-de-Quirós et al. (17), en España; y Miedema et al. (20), en Canadá.

Hay que mencionar, además, que el $80 \%$ los profesionales que participaron en este estudio reportaron haber recibido agresiones en más de una ocasión, cifras que están muy por debajo de las reportadas por Cervantes et al. (14) y Martínez et al. (12), quienes en sus estudios encontraron un $42,5 \%$ y $28,3 \%$, respectivamente, en 
cuanto a reincidencia de agresiones a estos profesionales.

En cuanto al tipo de agresor, los hallazgos de este estudio coindicen con los de Ancalli et al. (23), quienes manifiestan que el principal agresor es el familiar del paciente, pero difiere con los de Martínez et al. (12) y Lameiro et al. (16), quienes encontraron que son los pacientes mismos los principales agresores de los profesionales de la salud.

Por otra parte, al analizar las causas que dieron origen a la agresión, este estudio encontró que la principal de ellas es el retraso en la atención del paciente, seguida por el estado de alcoholismo o drogadicción del paciente y la no aceptación de las normas de la institución hospitalaria, hallazgos que coinciden con los de Martínez et al. (12), Lameiro et al. (16), Bernaldo-de-Quirós et al. (17) y Farias et al. (18).

Este estudio pudo evidenciar que las agresiones a los médicos y enfermeras en los servicios de urgencias de la red pública es una realidad frecuente, pero Colombia no es el único país que registra altos niveles de violencia contra estos profesionales, sino que es una práctica muy extendida y común en varios países del mundo, evidenciada por las investigaciones consultadas. La violencia contra los profesionales de la salud es inadmisible e injustificable, y las autoridades competentes deben tomar todas las medidas necesarias para protegerlos y no esperar hasta que los usuarios descarguen toda su frustración e irracionalidad contra quienes les están prestando un servicio.
La violencia contra los profesionales de la salud debe ser considerada como un problema grave, es por ello que en Colombia se debe elevar a agresión contra servidor público esta reprochable acción; para que quien la cometa responda ante las autoridades. Debemos rescatar y defender esta loable profesión de quienes se creen con la potestad de ofenderlos o agredirlos. Además, las instituciones prestadoras de servicios y las administradoras de riesgo laboral (ARL) deben establecer acciones, programas y estrategias para disminuir, minimizar o desaparecer las agresiones a que son sometidos los profesionales de la salud en el ámbito laboral y que atentan contra su dignidad, su integridad y sus derechos.

Es de anotar que el estudio presentó algunas limitaciones en cuanto al tamaño de la muestra y el haber sido realizado en instituciones públicas y en los servicios de urgencias, ya que como lo demuestra la literatura los profesionales de salud son agredidos en todos los servicios. Sería importante realizar nuevos estudios de este tipo en otros servicios y con otros tipos de profesionales de la salud que no fueron considerados en el presente estudio.

\section{Agradecimientos}

El autor expresa los agradecimientos a las enfermeras Diana Yohana Vargas Gualteros, Miryan Andrea Garzón, Íngrid Paola Torres Sánchez, de la Facultad de Enfermería de la Fundación Universitaria del Área Andina, por sus valiosos aportes a la investigación que dio origen al presente artículo, y al Dr. Bernardo Ortiz, por la revisión del resumen en inglés. 
Conflicto de intereses: Ninguno.

Fuentes de financiación: La presente investigación fue parcialmente finan- ciada por la Fundación Universitaria del Área Andina. Los demás gastos fueron asumidos por el investigador.

\section{Referencias}

1. Organización Internacional del Trabajo (OIT), Consejo internacional de enfermeras (CIE), Organización Mundial de la Salud (OMS), Internacional de Servicios Públicos (ISP). Directrices Marco para afrontar la violencia laboral en el sector salud. [Monografía en Internet]. Ginebra. 2002. [Acceso el 13 de agosto del 2015]. Disponible en: http://www.ilo.org/wcmsp5/ groups/public/--ed_dialogue/--sector/ documents/publication/wcms_160911.pdf

2. Mayorca I, Lucena $S$, Cortés $M$, Lucerna M. Violencia contra trabajadores sanitarios en un hospital de Andalucía. ¿Por qué hay agresiones no registradas? Med Segur Trab [Internet] 2013; 59 (231) 235-258.

3. Organización Internacional del Trabajo (OIT). Programa de Actividades Sectoriales. Repertorio de recomendaciones prácticas sobre la violencia en el lugar de trabajo en el sector de los servicios y medidas para combatirla. Ginebra. 2003. [Acceso el 30 de septiembre del 2015]. Disponible en: http://www.ilo.org/wcmsp5/ groups/public/_-ed_protect/--protrav/_-safework/documents/normativeinstrument/wcms_112578.pdf

4. Carrasco M, González J. Aspectos conceptuales de la agresión: definición y modelos explicativos. Acción Psicológica. 2006; 4(2):7-38.

5. Gascón S. Análisis médico-legal de la violencia en centros asistenciales: agresiones a profesionales. Tesis Doctoral.

Facultad de Medicina. Universidad de Zaragoza. 2006. [Acceso el 5 de octubre de
2015]. Disponible en: http://zaguan.unizar. es/record/5746/files/TESIS-2011-027.pdf

6. Organización Colegial de Enfermería. Consejo General. Estudio sobre agresiones a la profesión enfermería. [Monografía en Internet] 2012. [Acceso el 25 de octubre del 2015]. Disponible en: http:// www.index-f.com/lascasas/documentos/ Ic0446.pdf

7. Agreda J, Yanguas E. El paciente difícil: quién es y cómo manejarlo. An. Sist. Sanit. Navar [revista en Internet] 2001. [Acceso el 30 de Septiembre del 2015]; 24 (2). Disponible en: http://recyt.fecyt.es/ index.php/ASSN/article/view/5899/4754

8. Fawzi R, Hasan A. Physical Violence in the Workplace among Jordanian Hospital Nurses. Journal of Transcultural Nursing. 2011; 22(2):157-165.

9. Howerton R, Mentes J. Violence Against Women: The Phenomenon of Workplace Violence Against Nurses. Issues in Mental Health Nursing. 2010; 31:89-95.

10. Instituto Nacional para la Seguridad y Salud Ocupacional (NIOSH). Violencia: Peligros ocupacionales en los hospitales. DHHS (NIOSH). Publicación N. ${ }^{\circ}$ 2002101. 2002. [Acceso el 3 de noviembre de 2015]. Disponible en: http://www.cdc.gov/ spanish/niosh/docs/2002-101_sp/

11. Galián I, Llor B, Ruiz JA. Violencia de los usuarios hacia el personal de enfermería en los hospitales públicos de la región de Murcia. Rev Esp Salud Pública 2012; 86:279-291. 
12. Martínez B, Gascón S, Santed MA, Goicoechea J. Análisis médico-legal de las agresiones a profesionales sanitarios. Aproximación a una realidad silenciosa y a sus consecuencias para la salud. Med Clin. 2007; 128:307-310.

13. Altemir M. Agresiones al personal sanitario estrategias de afrontamiento de la conducta agresiva del paciente. Trabajo de grado. Facultad de enfermería. Universidad pública de Navarra. España. 2014. Pág. 4-34.

14. Cervantes G, Blanch J, Hermoso D. Violencia ocupacional contra profesionales sanitarios en Cataluña notificada por Internet (2007-2009). Arch Prev Riesgos Labor. 2010; 13(3):135-140.

15. Ortells N, Muñoz T, Paguina M, Morató I. Caracterización de las agresiones producidas al personal sanitario del servicio de urgencias en un hospital comarcal. Enferm. glob. 2013; 30:196-207.

16. Lameiro C, Besteiro A, Cuevas A, Pérez A, Gómez C, Del Campo V. Violencia laboral en Instituciones sanitarias; Análisis de un perfil cambiante. Enfermería del Trabajo 2013; III: 66-74.

17. Bernaldo-de-Quirós M, Labrador $F$, Piccini A, Gómez M, Cerdeira J. Violencia laboral en urgencias extrahospitalarias: una revisión sistemática y líneas de intervención psicológica. Clínica y Salud. 2014; 25:11-18.

18. Farías A, Sánchez J, Acevedo G. La violencia ocupacional en centros de atención primaria de la salud. Revista de Salud Pública. 2012; 1:18-26.

19. Travetto $C$, Daciuk N, Fernández $S$, Ortiz P, Mastandueno R, Prats M, et al. Agresiones hacia profesionales en el ámbito de la salud. Rev Panam Salud Pública. $2015 ; 38(4): 307.15$.
20. Miedema B, Easley J, Fortin P, Hamilton R, Tatemichi S. Disrespect, harassment, and abuse. All in a day's work for family physicians. Can Fam Physician. 2009; 55:279-85.

21. Winstanley S, Whittington R. Aggression towards health care staff in a UK general hospital: variation among professions and departments. J Clin Nurs. 2004; 13(1):3-10.

22. Kowalenko T, Walters BL, Khare RK. Workplace violence: a survey of emergency physicians in the state of Michigan. Ann Emerg Med. 2005; 46 (2):142-7.

23. Ancalli F, Cohaila G, Maquera-Afaray J.Agresiones contra el trabajador de salud en Tacna, Perú. Rev Peru Med Exp Salud Pública. 2012; 29 (3):415-6.

24. Tuya X, Mezones E. Violencia contra médicos: un problema por considerar en la investigación de recursos humanos en salud. Rev Peru Med Exp Salud Pública. 2012; 29(1):164.

25. Martínez M, Queipo D, Irurtia M, Martínez C. Análisis médico-legal de las agresiones a los profesionales sanitarios en Castilla y León (España). Rev Esp Med Legal. 2012; 38(1):5-10.

26. Diario El Colombiano. Junio 28 de 2015. Disponible en: http://www.elcolombiano.com/las-agresiones-a-personal-medico-van-en-aumento-MA2211790

27. Diario El Espectador. Febrero 24 de 2011. Disponible en: http://www.elespectador.com/noticias/bogota/secretaria-de-salud-preocupada-agresiones-personal-medi-articulo-252997

28. Diario El Tiempo. Julio 11 de 2014. Disponible en: http://www.eltiempo.com/ bogota/medicos-atacados-por-sus-pacientes-en-bogota/14233384 
INVESTIGACIONES ANDINA No. 34 Vol. 19

29. Diario $A D N$, diciembre de 2014. Disponible en: HTTP://DIARIOADN.CO/ MEDELLIN/MI-CIUDAD/AGRESIONESA-PERSONAL-M\%C3\%A9DICO-DELHOSPITAL-SAN-VICENTE-1.137532
30. Diario La Opinión. Junio de 2015. Disponible en: http://www.laopinion.com.co/ c-cuta/denuncian-agresiones-contra-personal-m-dico-93694 